\title{
Correction to: Multimethod investigation of the neurobiological basis of ADHD symptomatology in children aged 9-10: baseline data from the $A B C D$ study
}

Max M. Owens (1D, Nicholas Allgaier, Sage Hahn, DeKang Yuan, Matthew Albaugh, Shana Adise, Bader Chaarani, Joseph Ortigara, Anthony Juliano (1), Alexandra Potter and Hugh Garavan

Correction to: Translational Psychiatry https://doi.org/10.1038/s41398-020-01192-8

published online 18 January 2021

The original version of this article unfortunately contained a mistake. Due to a coding error, the final demographic table (Table 1) was reported incorrectly.
The correct Table 1 can be found below. The authors apologize for the mistake. The original article has been corrected.

Published online: 12 April 2021 
Table 1 Descriptive statistics on samples for each MRI paradigm.

\begin{tabular}{|c|c|c|c|c|c|c|c|c|}
\hline & \multicolumn{2}{|l|}{ sMRI } & \multicolumn{2}{|c|}{ EN-Back } & \multicolumn{2}{|l|}{ SST } & \multicolumn{2}{|l|}{ MID } \\
\hline & Mean & SD & Mean & SD & Mean & SD & Mean & SD \\
\hline \multicolumn{9}{|l|}{ Child age } \\
\hline \multirow[t]{2}{*}{ Months } & 119.18 & 7.46 & 119.61 & 7.53 & 119.53 & 7.54 & 119.42 & 7.52 \\
\hline & Total & $\%$ & Total & $\%$ & Total & $\%$ & Total & $\%$ \\
\hline \multicolumn{9}{|l|}{ Child sex } \\
\hline Female & 4179 & $49 \%$ & 2681 & $49 \%$ & 2524 & $50 \%$ & 2973 & $50 \%$ \\
\hline \multicolumn{9}{|l|}{ Child race } \\
\hline White & 5917 & $69 \%$ & 3878 & $72 \%$ & 3596 & $72 \%$ & 4174 & $70 \%$ \\
\hline Black & 1140 & $13 \%$ & 592 & $11 \%$ & 570 & $11 \%$ & 712 & $12 \%$ \\
\hline Asian & 177 & $2 \%$ & 111 & $2 \%$ & 105 & $2 \%$ & 118 & $2 \%$ \\
\hline Other & 1362 & $16 \%$ & 836 & $15 \%$ & 749 & $15 \%$ & 955 & $16 \%$ \\
\hline \multicolumn{9}{|l|}{ Child ethnicity } \\
\hline Hispanic & 1604 & $19 \%$ & 997 & $18 \%$ & 911 & $18 \%$ & 1122 & $19 \%$ \\
\hline \multicolumn{9}{|l|}{ Household income } \\
\hline$<\$ 50 \mathrm{~K}$ & 2303 & $27 \%$ & 1313 & $24 \%$ & 1228 & $24 \%$ & 1514 & $25 \%$ \\
\hline$\$ 50 K-\$ 100 K$ & 2474 & $29 \%$ & 1588 & $29 \%$ & 1462 & $29 \%$ & 1736 & $29 \%$ \\
\hline$>\$ 100 K$ & 3819 & $44 \%$ & 2516 & $46 \%$ & 2330 & $46 \%$ & 2709 & $45 \%$ \\
\hline \multicolumn{9}{|l|}{ Parent education } \\
\hline$<$ HS diploma & 283 & $3 \%$ & 142 & $3 \%$ & 136 & $3 \%$ & 169 & $3 \%$ \\
\hline HS diploma/GED & 644 & $7 \%$ & 352 & $6 \%$ & 338 & $7 \%$ & 421 & $7 \%$ \\
\hline Some college & 2153 & $25 \%$ & 1303 & $24 \%$ & 1197 & $24 \%$ & 1465 & $25 \%$ \\
\hline Bachelor & 2336 & $27 \%$ & 1547 & $29 \%$ & 1434 & $29 \%$ & 1675 & $28 \%$ \\
\hline Post graduate degree & 3180 & $37 \%$ & 2073 & $38 \%$ & 1915 & $38 \%$ & 2229 & $37 \%$ \\
\hline
\end{tabular}

